\title{
[Closing Remarks]
}

1. K. Boyex

We see the difference between the situation of today's conference and the previous one which professor Yamanaka has sponsered. Now laser is becoming available and you can do useful experiments with it. I think not only at $1.06 \mu$ range but also at the range from $10.6 \mu$ down to a quater of micron, it is very important to continue the interaction studies and to get good understanding of both interaction and energy transport processes and this kind of work was only begun.

I think the compression experiment has been done very encouragingly but they are not yet at the stage where we expect to run real stability and symmetry. One of the tasks next several years for the experimenters is doing the experiments of the compression and explosion and looking at the symmetry. Another is taking more sophisticated instrumentation and we actually have no operation at LLL and our laboratory. I'm sure that such a thing is time resolved $\mathrm{X}$-ray photography for this type of experiments.

Now recently something has happened and the great helps to the laser fusion program is the change of classification policy in U.S. Many thing we are talking about now could not be known in both LLL and Los Alamos until this time. I think that not only exchange of information but also next two things are useful. One is the cords which have been developed paciffically for the laser fusion and these cords will be possibly made avairable. Some of them are required considerable computation capacity to use and I think that will be one problem but I think getting some of these cords will be very useful.

The other is a pellet fablication and the more sophisticateed pellet. We would be interested in furnishing such pellet in exchange for the data where other laboratories have real capability for the irradiating such pellets. I am glad to show to people the techniques for making there various pellets which is very similar in Los Alamos and LLI and I think this will be very useful.

Now I think there is the prospects for laser fusion still look quite right to me. We see the problems but we have not yet run into anything which we can not make work in fundamental way. I think it is important to continue the experiment so that we can determine what reality of these various theoretical prediction are. The things which we are now observing in the interaction region are quite different from that were forecast seven years ago purely in theory.

\section{M.J. Lubin}

I would like to say three points. No.1 will be optimistic. I think that within next few years,one or more facilities around the world probably make significant neutron yield. The significant neutron yield I mean is in excess of $10^{12}$ neutrons per shot and beyond this value the scaling law will be successful. 
Second, it is the last and most ideal and unfortunately most difficult to make econimic neutrons for fusion power. This is a long range problem and to make use of energy directly is even long range problem.

The last point that $I$ want to make concerns is that the science of absorption, reflection and some of physics come out even in this meeting, I made a table on which some of comments on reflectivity measurements, presence of fast ions and intensities from various laboratories. I can see in this table any particular term, that is the presence of fast ion components which may have something to do with the orientation of $\mathrm{k}$ vector on target and the regeneration of self-induced magnetic field which will prevent a radiothermo-transport into dense core but $I$ don' $t$ know in detail. At any rate these parameters written down here is to look at for both experimentalist and theoretical people and I am sure that all these experimental data are open to your suggestion.

I look forward to work on much more closely with laboratories around the world.

\section{P.K. Kaw}

I think that the topics which will be discussed could be classified into three main topics.

One is just the laser-plasma interactions in which are discussed absorptions, scattering, deflection, caviton and everything. The next topic is about the energy transport which include the topic of fast ion production and reduced thermal conductivity which takes reduced energy flow inside the system. And the third topic will be compression and associated instability.

In the laser plasma interaction, it seems to me that as if Moose pointed out this note definitly, one thing is that absorption is about 30 to $50 \%$ or more. Another thing which seems to come out of Moose's experiments, is that absorption is independent of power over very wide power range. This is the very interesting result. The second point is the reflection seems to go down to about $10 \%$ when the power is very high. The third point is the refraction is about 60 to $70 \%$. So if one can believe this kind of break up of the energy, $30 \%$ seems to go into the pellet, 108 seems to go as a reflected power, and $60 \%$ is going around the pellet in the case of the spherical target.

I think that one needs the energy balance instantaneously for the entired build up the pulse. One is to looked at the reflection, transmission and scattering at all the time from the beginning to the end of the pulse, so that you can see where is the laser energy going at each instant of time. If we understand as what fraction of laser energy is really getting absorbed and what is the mechanism of the absorption, only we can hope to improve the fraction to go to $30 \%$ to $70 \%$ and to $100 \%$, but really understand the laser plasma interaction. I think it is very important that we get the energy balance in a time resolved manner. I try to understand at each instant 
of time to get energy balance equation.

Why is the absorption independent of power? Is it because of no parametric instability or is it because if linear mechanism ? Does the absorption modified overbear the effect of parametric instability?

Another things, which seem to come out to discussions, were profile modification near critical layer, laser crucial law in the absorption process, the caviton formation for many experiments, the self-phase modulation discussed by Yamanaka and the after pulse discussed by Lubin. Kruer presented by the computer simulation, the strong caviton formation and the very strong modification of density profile near the critical layer. Now there is some questions about whether the caviton is only one dimension or two or three dimensions. Perhaps we have two or three dimensions if you balance nonlinearity, damping and dispersion. It seems not to be the theoretical imagination.

Why is the reflection so low at high powers, this is a main question. I think we have no answer today. But one possibility is the turbulence could reduce the reflected energy and backscattering.

Next topic is the energy transport. Here the different experimental peoples do not agree on their results, but I found only conclusion of fast ion production coming from Los Alamos group. The question is under what conditions do you get the fast ions and also the relaxation of thermal conductivity. It seems to me that whole problem of preheat has been reversed and everybody will know the fact that energy will be deposited at the surface and carried to the center too soon. The macroscopic calculation done by Los Alamos is very interesting: They introduced the flux factor. It can give you fast accelerated ions because of trapped electrons and also a reduced transport of energy inwards. But in the microscopic picture, the density fluctuation that they need for getting flux limitaion factor will be about 0.1 or 0.3 of $50 \%$ or $100 \%$. This is a very large density fluctuation. I get the reason for this that the magnetic field has a very crucical law for the transport of energy. So what has to be done in calculation is a combination of turbulence, turbulent transport in the magnetic field. If it is feasible to think a special pellet design and special material, you create any situation of energy deposition, production of enhanced lamdau damping by ion waves and the energy flow to the point you want to take or to the interior.

About the compression and instability problems, the nonlinear fate of instability was the only experimental question. I think the calculation should concentrate on that what kind of find state comes from instability, what has the layer nonlinear level and what is the consequence as far as the experimented observation concerned.

\section{C. Yamanaka}

I would like to conclude the session sumarizing the following points which are essentially important to approach the laser fusion goal. 
(i) The development of the high power lasers

To understand the developed, the developing and the under developed conditions of the laser, we shall have a technical review concerning the state of the laser arts at least the detail table of the present lasers in operation. The efforts to persuit the new lasers should be encouraged by the international interests.

(ii) Interaction physics between laser and plasma

The basic research on the laser interation is very important not only from the academic interest but also to build up the big implosion scheme. Still there are many problems to be solved. The self-focussing and caviton model are now on the attention after the fasion of the parametric absorption and stimulated scattering. This new situation is well investigated by the self-phase modulation of the laser light due to the density change in plasma and the microwave simulation and computers. -Doping by high $z$ matirials and also the suitable laser pulse concerning the intensity, the duration, the form, the precurser should be investigated to prevent the appearance of the energetic electron and the fast ion.

Diagnostics are also very important, especially they need high spacial and temporal resolution which are not in ready made. I would like to propose the international discussion of work. The mutual correspondence on the different approachs will promote the quick advance in research. And also we should try to recognize a same items. As the existence of the fast and the slow ion at the laser power beyond a threshold of the parametric instability is now approved by several laboratories. The reflectivity, the distribution of the laser energy and so on. (iii) Implosion, SFX and etc.

Now I bilieve the laser fusion comes up to the second stage at this time. The implosion experiment, even in a preliminary state, has been performed succesfully at KMSF. This is very encourageous event to all laser people inspite of the several critisisms from outside and inside. The computational experiments are really expected to approach the results of the physical experiments. They were so often optional and present the contradicted results, as will known, on the stability problem of the implosion.

Before the SFX of the laser fusion, we would like to have the intermediate objects, such as neutron source, a X-ray source which are very interesting scientific tools. And also the isotope separation!! These might be strong supports to keep on the long way to the laser fusion goal. However, the laser fusion, I believe, will be attained not later than the magnetic confinement schemes.

(iv) Technical developments

The fabrication of the targets, the collimation of the

laser beams and several tedious technical problems are very important to make a real advance to the unknown. We have succeeded to develop a laser glass, U.S.A. has recently developed a microballon target.

Really these technical developments are definit steps to the future, on which the whole scheme depends. Much more efforts shall be devoted.

At the end of the remarks I propose to keep the close 
contacts each other on the above mentioned points to exchange the data and to cooperate for the promotion of the research front as soon as possible to get the nuclear fusion for the human kind.

At last I express my sincere thanks to the all participants who kindly arrange to attend the Fuji Seminar just prior the 5 th IAEA meeting. 


\begin{tabular}{|c|c|c|c|c|c|c|c|c|c|c|}
\hline $\begin{array}{l}\text { 彳 } \\
\text { है } \\
8\end{array}$ & 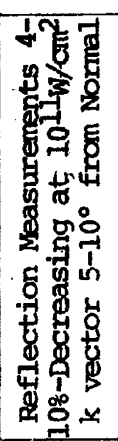 & 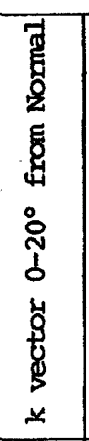 & 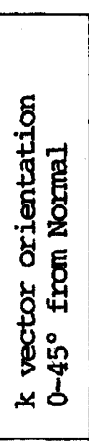 & 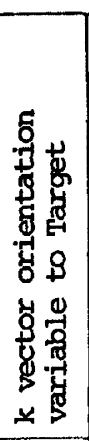 & 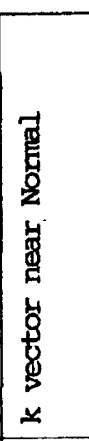 & 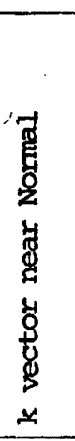 & 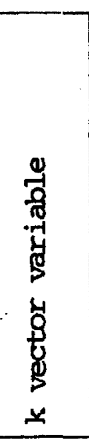 & & 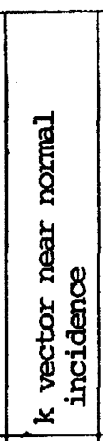 & 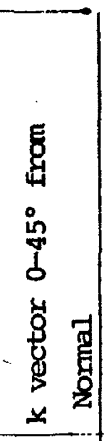 \\
\hline 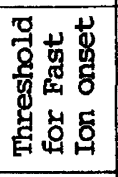 & & नै० & $\because$ & $\nRightarrow$ & $\stackrel{I}{\circ}$ & $\overbrace{0}$ & 焉 & & 1 & \\
\hline 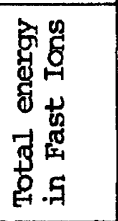 & & $\stackrel{\infty}{\infty}$ & : & $\stackrel{d o}{\stackrel{d}{v}}$ & 。्p & 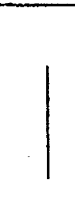 & $\stackrel{\text { ڤొ }}{\circ}$ & & 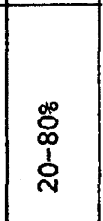 & $\stackrel{\text { ํํㅁ }}{v}$ \\
\hline 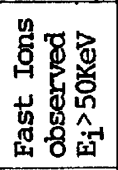 & $\stackrel{\circ}{\circ}$ & 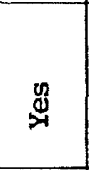 & $\coprod_{\nsim}^{\mathbb{N}}$ & no. & $\underset{x}{\stackrel{y}{y}}$ & $\stackrel{\mathscr{W}}{\not}$ & 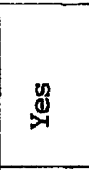 & & $\underset{x}{\mathscr{y}}$ & 8 \\
\hline 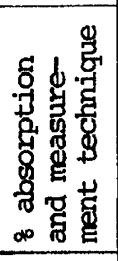 & 1 & 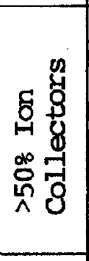 & 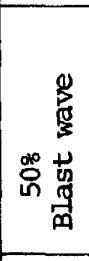 & 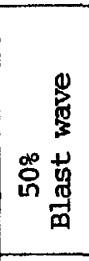 & 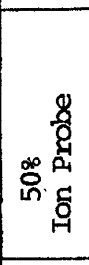 & 哭 & 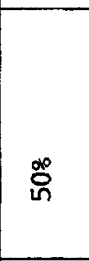 & 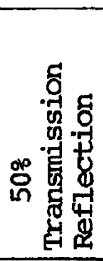 & 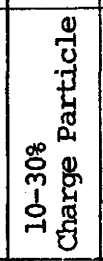 & 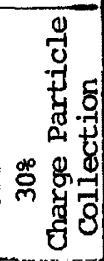 \\
\hline 总总 & 1 & 1 & ᄅ् & $y_{y=}^{g}$ & 1 & 1 & $\bigcup_{x=1}^{\infty}$ & 1 & 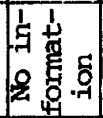 & $\sum_{0=1}^{\infty} \&$ \\
\hline 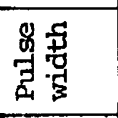 & हू & 苞 & 总 & 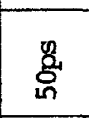 & 迎 & בू & 罡 & め & 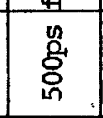 & 㖞 \\
\hline 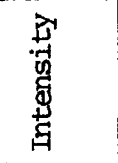 & $\overbrace{0}$ & 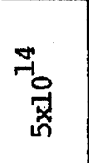 & 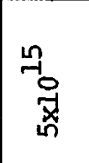 & $\stackrel{n}{-10}$ & 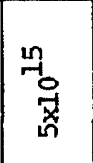 & 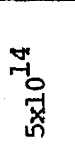 & $\stackrel{2}{\circ}$ & 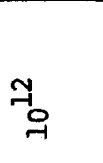 & ?口 & ?: \\
\hline 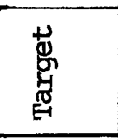 & శ్రీల్ & 题 & 营 $\tilde{\theta}^{N}$ & 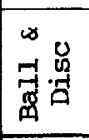 & 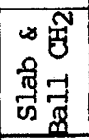 & जี & శ్ & $\begin{array}{l}7 \\
\overline{7} \\
8\end{array}$ & 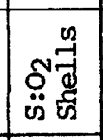 & 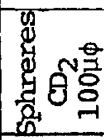 \\
\hline 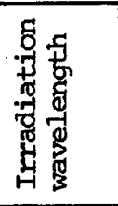 & $\begin{array}{l}\overrightarrow{7} \\
\dot{0} \\
\dot{\sigma}\end{array}$ & $\begin{array}{l}7 \\
\vdots \\
\dot{9}\end{array}$ & 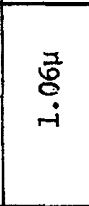 & ثَّ & $\underset{\text { ì }}{\stackrel{7}{\circ}}$ & $\begin{array}{l}7 \\
\vdots \\
\dot{9}\end{array}$ & $\underset{-i}{\stackrel{7}{\circ}}$ & $\begin{array}{l}7 \\
\text { 응 } \\
0\end{array}$ & 형 & -i \\
\hline $\begin{array}{l}8 \\
9 \\
9 \\
8 \\
9\end{array}$ & 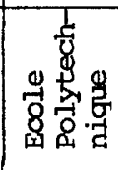 & 㩆密 & & & $\begin{array}{l}\text { 嗬 } \\
\text { 尊 }\end{array}$ & & 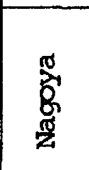 & 害 & 忽 & 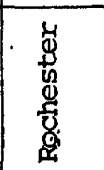 \\
\hline
\end{tabular}

